This is a draft of a chapter that has been accepted for publication by Oxford University Press in the forthcoming book Oxford Handbook of Acceptance and Commitment Therapy edited by Michael P. Twohig, Michael E. Levin, \& Julie M. Petersen due for publication in 2021-2022.

\title{
Clinical behavior analysis and RFT: Conceptualizing psychopathology and its treatment
}

\section{Carmen Luciano, Niklas Törneke, and Francisco J. Ruiz}

\section{Introduction}

In the night of November $27^{\text {th }}, 1994$, the cruise ferry MS Estonia sank in the Baltic Sea on its way from Tallinn to Stockholm. There were 989 people were on board; 852 people died in the disaster and 137 survived. The rescue efforts were dramatic, given the rough weather. The wind was 15-20 meters per second, and the temperature in the water was around 10 degrees Celcius. The ship sank quickly and the survivors were all rescued from rafts or directly from the cold water.

When survivors of the disaster describe their life experiences after the traumatic event, their testimonies confirm what we have learned from people who have had other life-threatening and extremely aversive experiences. Survivors describe a variety of effects following a trauma. Some of those seem more directly related to the events the survivors have been through. Memories of what happened and new situations of danger tend to trigger these memories and similar emotional responses. For instance, one person who survived the disaster of MS Estonia describes a turbulent air flight and how she was brought back to the experience of that night on the Baltic Sea. Other experiences can seem a bit more surprising, as one person who suddenly became very upset and anxious watching a dancing performance several years after the disaster. Only after a while, she realized that the way the dancers moved their hands reminded her of the ways people had waved that night several years back, sitting in rafts on the stormy sea. Still, in these examples, the connection between the traumatic event and things that occur later is pretty straightforward.

In addition, there is one more thing that people continuously report after traumatic life events: they have to face their own, most commonly aversive, reactions. There is a threat that comes from within, so to speak. This is also true about specific memories or other more complex reactions. For example, it might be the experience of life lacking meaning. One relatively common testimony is a disabling sense of guilt that is not connected to a particular behavior during the catastrophic event but to the very fact that the person survived. Some people, quite the contrary, report an increased sense of meaning that they can use to redirect their life, even in the presence of painful memories.

\section{Behavior analysis and private responses}

In the previous examples, the starting point is an extreme, aversive event. However, in many ways, the aversive event only highlights what is true more generally; human beings interact not only with the outward environment but also with their own responses, including those that are only detectable by the person emitting them (e.g., emotions, sensations, and thoughts). Furthermore, they do that in the context of the particular history of interactions with the world. There is an environment "within the skin," to quote a term used by Skinner. This is central to the human experience; it has long been generally acknowledged within behavior analysis as the self. Skinner (1953) used several chapters of his book, Science and Human Behavior, on the subject. He analyzed these phenomena and how we tend to interact with them under the heading of terms like "self-control," "self-knowledge," "self-awareness," or simply just "self." His classical term for responses of this kind, only detectable for the person emitting them, is "private events." Skinner's main argument in his analysis is that these phenomena should be understood as other behavior and not as occurring in some other mental sphere, like what is traditionally called "the psyche." However, Skinner's approach to these topics was somewhat interpretative, and the influence of this behavior was an unknown path even though prominent members of the behavior analytic 
community have long argued for their relevance (Day, 1971; Ferster, 1972).

\subsection{The position of clinical behavior analysis}

Even though the theoretical position taken by modern models of therapy like acceptance and commitment therapy (ACT), behavioral activation, functional analytic psychotherapy, and dialectical behavior therapy (DBT) is firmly rooted in the behavior analytic tradition, they share with psychotherapy the general assumption of the importance of private events for understanding and helping clients with psychological problems. The reason for this is in part practical and, in a way, the result of common sense. It is hard to imagine a dialogue with a person surviving a disaster like the one described at the start of this chapter and claiming that whatever the person remembers, feels, or thinks about the events is not relevant for therapy. The same is true for a more circumscribed problem, like a specific phobic reaction (e.g., encountering a spider). Claiming that it is all about the spider and that what the person feels, thinks, or senses in the interaction with the spider is irrelevant is a hard position to take. The same goes for an everyday situation of meeting someone in the street and then recognizing the person as an old friend. The possible claim that the behavior we label "recognizing" is irrelevant for analyzing the subsequent behavior seems to miss something essential. Still, the behavior of recognizing could well be private in the Skinnerian sense-not detectable to anyone except for the person behaving that way.

At this point of our argument, it is essential to note that, although we claim that private events might have an important function for further behavior, this is not to give these private behaviors a causal role in a mechanistic sense. What is claimed is that they can have a function, even an important one, in a sequence of behavior. Consequently, private events often should be targeted, one way or another, in an effort to help the person to change in a personally desired direction. In taking this position, we start at the point where Skinner stopped because he lacked the scientific data now available to continue his analysis. The importance of private events for a behavior analysis of human behavior is not only to affirm the relevant role that these events can have. It is to understand the context under which they are developed as well as what functions these kinds of events can have. And furthermore, it is yet another task to show how we can help people direct their lives the way they want to by using our analysis.

\subsection{Analyzing the function of private events with the help of RFT}

Basic, experimental science regarding human language and cognition has advanced since Skinner attempted to analyze these phenomena. One specific line of research that keeps the connection with the Skinnerian tradition of science is relational frame theory (RFT; Hayes, Barnes-Holmes, \& Roche, 2001). The key element of the findings presented in RFT is that the basic unit of human language is a specific repertoire of relating that humans learn in early childhood. This acquired ability then has enormous effects on human behavior overall. Language-able humans will interact with the environment according to how they have learned to relate one phenomenon to another in very complex ways. Alternatively, in a more specific behavioranalytic terminology, stimuli can acquire function in a way that is different from what has been demonstrated for non-human animals due to the learned ability to relate in this particular way. The broad findings of RFT are covered in Chapter 4. At the same time, a few specific lines, findings, and conclusions from this research need to be discussed to take on the task of a clinical analysis-first, a general conclusion.

Once a human has learned the particular type of relating at the core of language, in RFT termed relational framing or arbitrarily applicable relational responding, all human interaction with the environment is affected by this ability. Events of all kinds acquire particular meaning to humans, potentially everything a human encounters is symbolic due to the ability to frame relationally. However, the specific meaning is contextually controlled by the person's history and the present circumstances

The characteristics of relational framing are not restricted, though, to events in the physical environment. The same holds for events that are aspects of the behavior of the human, what the tradition of behavior analysis has termed private events. Feelings, memories, thoughts, and bodily sensations are developed and acquire complex functions due to relational framing and, thus, influence behavior in critical ways. We can now go 
beyond the position where the reason for assuming the importance of private events for human functioning is common sense-it is also empirical. RFT provides us with tools to analyze how private events develop and acquire such complex functions, how different ways of interacting with private responses develop and lead to behavioral traps, and how we can assist clients in overcoming such traps and redirecting their lives. To do such an analysis, we need to go somewhat into depth with particular aspects of how we learn to interact with our own responding and what type of behavior this is. This is the phenomenological area Skinner referred to as "the self." As the self is not an object but a specific type of complex human behavior, we will use the term "selfing."

\section{The behavior of selfing}

Selfing behavior is at the core of the human condition: it is a sophisticated, learned behavior that makes human beings conscious of themselves as acting organisms. We learn to respond to our own behavior and thus learn to make choices and be conscious of that behavior and all that follows. This type of responding might be effective or ineffective to our living, with the latter being the source of psychological problems. Being so central, one of the most relevant lines of research from a behavior analytic point of view is to identify the conditions under which we learn selfing behavior (e.g., emotions, thoughts, memories), as well as how this behavior becomes a source of functional control for subsequent behavior. Said in another way, thoughts and connected emotions influence subsequent responding. Again, this does not mean that one behavior is a formal cause for another behavior. However, private events acquire a functional role due to a particular person's learning history. That is, the history in the present circumstances brings the function for the actual responding and the respective consequences. These events are then the whole behavioral universe at any given moment.

This section is organized into three parts. First, we will point to early behaviors that contribute to building the repertoire of selfing, especially the early conditions establishing that one behavior acquires functions for another. Secondly, we will point to the impact of learning to relate and the transformation of antecedents and consequences. Thirdly, we will point to how deictic and hierarchical responding account for the sophisticated selfing repertoire. Finally, we will emphasize how two functional classes of responding to the one's own behavior are developed: one flexible and effective repertoire and the other inflexible and ineffective.

\subsection{Early building of self-behaviors}

As we know, human beings respond to the functions of present circumstances. The functions are generated from the phylogenetic background and the ontogenetic learning history of interactions with the physical and social systems. This is the whole. Consequently, the background of the selfing behaviors is the history with the verbal community. As Skinner (1974) pointed, this means that others' behavior functions either as consequences or antecedents of the individual's response. This interplay between your own behavior and the behavior of others is central for learning the repertoire of being conscious of yourself as a unique human being and being in charge of your own actions. On this basis, the contingencies provided by the verbal community are the context for learning also private behavior. Even behavior that is not directly accessible to others is built on interactions with others (Skinner, 1945). So, the reaction of others to your responding becomes the main context for learning essential behaviors that characterize human beings, such as knowing who you are, what you want, where you are, what you are doing, why you are doing this, or even more, the you who realize who is thinking, who is doing, who is giving reasons or noticing what happens because of some reason, etcetera (Hayes, 1984). How does all this happen?

\subsubsection{Early interactions of responding to others'} behavior. The history of an individual begins within the inevitable interactions with others as the core context for development. The explicit contingencies under which we learn to respond to questions and instructions given by others are the key context for developing the motivations that will be central in our behavior. That is, at the same time the babies learn to name objects and events according to the behavior of others, they also learn to follow instructions under specific consequences, resulting in learning either to enjoy or to avoid those consequences. All of this is according to the cultural contingencies in the environment. For instance, the consequences might select responding based on the mediation of others or might select responding based on the natural consequences of the behavior. These two types of 
consequences are critical in forming the operants as classes of behavior that, later on, will be the basis upon which to build our way of being in the world.

In this process, the children are asked for formulations about who, what, and why they do what they do, did, or will do; and they respond in the context of others prompting and reinforcing answers. Of course, the others behave according to the coherence given as a mirror of their own history. The opportunity to respond and the reinforcing consequences the others provide establish the repertoire needed for the child to develop the sophisticated selfing behavior. Then, the building of the different selfing behaviors is directly connected with the conditions provided by the others, either for following instructions and for deriving rules, and subsequently will control specific actions. These early contingencies of the multiple-exemplar training (MET) will build functional classes that will become established under the meaning, interests, and values, as higher-order or hierarchical motivational functions controlling the own behavior of the individual.

More precisely, early on, the child is taught to follow instructions under the control of the consequences mediated by others (e.g., "Good that you are doing what I ask you to do. I am very glad and I will permit you to play outside.") When MET of these behaviors are in place in a dominant way, then the children are taught to attend more to other's behaviors as the main source of motivation (be positive or negative reinforcement) than to other consequences (e.g., the direct effect of their behavior beyond their parents's attention). However, it might happen that the children are taught to pay attention to what happens in the environment as a function of their behavior, that is, to look for the changes occurring because of their behavior (e.g., "Look, because you are wearing your coat, you are warm in this cold and windy day.") When MET of these behaviors is in place in a dominant way, the child's behavior becomes motivated for those natural consequences without the mediation of others' behavior, again, this being effective or ineffective depending on the context.

So, MET might result in (a) a pattern of responding, as a generalized operant selected under the consequences based on the mediation of others' behavior. This responding can be based on positive or negative reinforcement as receiving attention per se, or receiving attention to achieve subsequent positive consequences or to escape/avoid from some aversive functions. It might result in (b) a pattern of responding, as a generalized operant selected under the natural consequences of responding, that is, according to how the physical and social world works, either based on positive or negative reinforcement. Moreover, the MET of these behaviors might form functional classes under the control of continuous or intermittent consequences. Even more, for a particular person, the contingencies in the MET might have established only one pattern of any of these two functional classes. In contrast, for another person, the contingencies in the MET might have developed functional classes appropriately contextualized to both the survival contingencies in the natural and the social worlds. An example of the latter case should be having learned to respond under the control of the others' behavior in particular circumstances and under the control of the natural consequences of his behavior in other circumstances. However, this is not the only story. All that means that it is the individual what matters and, consequently, that what is important is to check for the specific personal conditions.

At the same time the child is learning to follow simple instructions and these functional classes are being built, the child is also learning to frame relationally under specific functions. Learning to frame relationally is a before-after point in the child's development. It is crossing the Rubicon, a crossing that will dress the previously learned functional classes with the coherent function attached to learning the behavior of arbitrarily applicable relational responding. This is there where we move our attention in the next section.

\subsection{Learning to relate and derive relations and functions}

According to RFT, relational framing underlies human language and cognition. Relational framing means relating two or more events based on arbitrarily established contextual cues (Hayes et al., 2001) such as coordination ("is," "same as," "goes with," etc.), opposition ("opposite to," "is the contrary of"), distinction ("different from"), comparison ("more than," "less than"), spatial ("below," "above," "inside," "outside," etc.), hierarchy ("belongs to," "includes," "part of"), temporal ("before," "after"), causal ("if... then") and deictic (I-You, Here-There, Now-Then). All types of relational framing are generalized operant behaviors that are typically learned early in life through METs 
provided by the verbal community. Importantly, learning to relate this way permits deriving new relations without direct training and that the functions of one stimulus transform the functions of the stimuli related to it according to the specific type of relations. This is the core characteristic of relational framing that accounts for the generativity of human behavior. Examples of the properties and types of relational framings are outlined in the following paragraphs (Hayes et al., 2001; see the chapter in this book)

The different patterns of relational framing differ in relational complexity so that some seem to develop before others. For instance, after developing more complex repertoires of relational framing, Susan, our example child, shows more sophisticated behavior. For example, when learning to derive relations according to comparison, opposition, and deictic, she can understand her father saying, "Sweden is a cold country, but Spain is the opposite" by deriving that Spain is a hot country. Further, because of the learned opposite relation between being right and wrong, and being right as being intelligent and loved by others, when making a mistake, she begins to derive "Susan is silly and is not going to be loved." This derivation makes her feel sad and the urge to respond correctly. Even more, she begins to count the number of times that she and her schoolmates Violet and Joe responded correctly to the math teacher's questions and derives, "I am smarter than Joe, but Violet is smarter than me, so Violet is the smartest one." According to this derivation, Susan feels confident and wants to compete against Joe doing math but avoids competing against Violet.

RFT defines two forms of contextual control over relational framing. The first form is called relational context $\left(\mathrm{C}_{\text {rel }}\right)$ and specifies the type of relation established among the stimuli. The $\mathrm{C}_{\text {rels }}$ usually are some of the arbitrary relational cues presented above, such as "is," "same as," "is the opposite to," "more than," "less than," "contains," etcetera. The second form of contextual control is called functional context $\left(\mathrm{C}_{\text {func }}\right)$ and specifies what functions will be transformed in a given moment. Following the latter example, Violet, Joe, and Susan were related in comparison regarding math skills. However, Susan has also learned that Joe is a very good soccer player, but Violet has no soccer skills. Thus, Susan has derived an opposite relation between Joe and Violet regarding soccer skills. However, when Susan's teacher asks her, "What teammate do you choose for your maths team?" the word "maths" works as the $\mathrm{C}_{\text {func }}$ to select Violet because she wants to win the competition. Alternatively, when asked to choose a teammate for a soccer competition, the word "soccer" works as the $\mathrm{C}_{\text {func }}$ to select Joe. Given these interactions, Susan is learning to derive with flexibility in relation to Violet, Joe, and herself.

Individuals differ in the opportunities for derived relational framing (Hayes et al., 2001; Luciano et al., 2009). In other words, a person might become more fluent deriving in a particular framing than others, with more or less contextual control as in Susan`s abovementioned examples. Each personal history is different, which implies that what might be a complex derivation for a person might be an easy one for another. Taking this into account, derived relational responding qualifies for a number of formal dimensions with different levels that have to be considered with caution because, in a functional analytic perspective, individual history is what matters (Barnes-Holmes, Barnes-Holmes, Luciano, \& McEnteggart, 2017). For instance, relational responding might show different levels of: (a) derivation (e.g., only the first time a derived response is produced qualifies as a fully derived response because this derived response contacts to the contingencies connected to the history of derived responding. Moreover, when the derived response is further repeated, it strengthens the network to which they belongs). At the same time opportunities to derive form the fluency in relational responding (e.g., more in deictic I-You than in deictic I-Then-before or more in I-Then-before than I-Here-Now), (b) complexity (e.g., given $\mathrm{A}>\mathrm{B}=\mathrm{C}$, deriving $\mathrm{A}$ is more than $\mathrm{C}$ is considered a more complex relational response than deriving $\mathrm{A}$ is the same as $\mathrm{C}$ given $\mathrm{A}=\mathrm{B}=\mathrm{C}$; deriving relations among relations, as in analogies, is considered more complex than deriving relations in one network or group of stimuli relations); (c) flexibility (e.g., contrary to the example of Susan with Joe and Violet, it is when a network has not been sufficiently contextualized. Then, responding will not be sensitive to contextual changes and the person showing such a network will not be easily adapted to the changes in contingencies, for instance).

Besides these formal dimensions (that might be used for filtering each particular history), derived relational responding shows a characteristic that is the very essence of this human ability: coherence. 
For Barnes-Holmes, Hayes, Dymond, \& O`Hora (2001), "coherence or sense-making appears to function as powerful reinforcer for relational activity" (p. 70); for Hayes, Stroshal, \& Wilson (2012), and Luciano (2017), coherence becomes the reinforcing context that envelops any instance of relational responding. Accordingly, coherence becomes a motivational context built across the opportunities to derive (Luciano et al., 2009), and it becomes the strenght of derived relational responding. Thus, derived relational responding only occurs in the context of coherence, that is, according to the particular history of relational framing.

Consequently, all that verbal organisms do show the coherence of their relational history. Having said that, a person might show an incoherent response from our perspective, but it is coherent according to the individual's history. Even when the present circumstances lets a person, Susan in our example, derive that "what is happening to me is not what it should be," or "I have been struggling and things are getting worse, I can not understand why, this is incoherent for me," none of these responses are incoherent with her over all relational responding. Quite the contrary, even when Susan says that something is incoherent, this is derived, or repeated, according to her particular coherent history of framing. That is, her history of relating permits her to frame what it should be occurring to her now as different -or opposite- to what is actually occurring to her. And because the former network is strength over the latter, according to her relational history, Susan derived thoughts and feelings as, "it can not be that way and I am very disappointed."

In other words, the strength of the our relational history is the coherence of our relational history. It is the same thing as when we respond to what is inconsistent with our own coherent history by trying to uphold the very coherence. For instance, trying to convey the others or ourselves that "we are right and the others do not understand anything" as a way to recover the consistency and reducing the discomfort associated with contacting the inconsistency between what happens and the ideas/rules established in the our history. Thus, the coherence of derived relational responding is present in all the selfing behavior as it is in any other relational responding. This is why coherence becomes a hierarchical function over any other characteristic or dimension of relational responding (Luciano, 2017).

\subsection{Deictic and hierarchical as the core framings in selfing behavior}

Deictic and hierarchical framing is at the core of most complex human behaviors. Both types of behaviors are essential for organizing coherent behavior overall and needed for responding to others and the relation between different aspects of one's behavior. As a whole, flexible deictic and hierarchical relational framing likely facilitates problem-solving and interpersonal skills

Deictic framing is learning to behave from different perspectives. Briefly stated, when the deictic framing repertoire is in place, the child can frame agent (I versus other), time (now versus then), and space (here versus there) from the unique I-herenow perspective. The social contingencies facilitating the distinction I-you are established early, and more complex contingencies are needed to establish I-now, I-before, I-after, I-here, I-there, and the respective combinations while learning to do it without losing the perspective of I-here-now (Barnes-Holmes, Hayes, \& Dymond, 2001; Luciano, Valdivia, Cabello, \& Hernández, 2009; Luciano et al.,, 2020).

Hierarchical framing is trained across many examples with different arrays of functional combinations so that different stimuli might be hierarchically organized. For instance, when the child is taught to respond to different stimuli (e.g., different cats, dogs, elephants, eagles, etc.) as belonging to a higher-order class based on sharing common functions (e.g., animals because of specific living functions). Hierarchical framing is perhaps the most sophisticated and flexible of the relational frames we can learn because of the flexibility to organize stimuli at different levels under different contextual functions. Social questions and contingencies are needed to establish the myriad of flexible organizations of stimuli. The kinds of hierarchical networks that might be established are numerous and they are essential to acquire flexibility in many different ways. To illustrate this, imagine the many ways you can organize animals, minerals, stars, humans beings, food, races, as well as organizations such as religions, political parties, countries, business, science, and even the taxonomic system of mental disorders, or the organization of a person's behaviors as types of personalities.

It is these latter examples that are of special interest in this chapter. First, we will see how all framings partake in developing private events as thoughts/ideas/actions about ME and OTHERS. 
Then, we will see how the abstraction of ME functions as a continuity or common experience across multiple behaviors of the same individual. Following, we will describe the functional hierarchical organization of these behaviors. Finally, we will describe two ways of responding (two functional classes) to these behaviors. One is responding in coordination with the function of one's own behavior in a way that opposes other functions, resulting in limiting consequences to your life. The other is responding hierarchically to one's own behavior, thereby integrating any other function of that behavior under the domination of another function, resulting in an adaptation to life. Let's explain this by going to the grounds, having described the surface.

\subsubsection{Deictic and hierarchical responding for deriving self-contents and establishing relational functional operants}

As with other stimuli functions, human beings respond to ongoing private events according to the personal history and contact the consequences of such responding. However, private events are not isolated phenomena, they are inevitably related to other private events in the person's history. Consequently, an ongoing private event and its relations to others events, the response to its functions, and the consequences contacted are a single unit of behavior. Through MET, instances of such units become related based on their functional properties and form relational functional classes around the experience named I/ME, with deictic and hierarchical framing at the core.

Let's present several scenarios of possible early interactions with a small child, Mario, and the people around him. This example is in some ways extreme but is given to illustrate how relational framing can build overarching functional classes of behavior. The same principles of learning are assumed to be present in less extreme experiences.

Watch Mario as he cuts his hand with a blue knife. His mother cures the wound and blames him for what happened. She scolds him because he did not pay attention and compares his behavior to his father's, who died some time ago in a car accident. After this, Mario starts to avoid knives and other physically similar objects (e.g., scissors). He refuses to pick up such things and open the kitchen drawer where spoons, forks, and knives are set. His mother also insists that he should wait for her to help him do certain things and instructs him to be as careful as his intelligent brother. After hearing his mother's description of the event ("It's because you did not take care, you did that dangerous thing and you got hurt"), Mario derived contents such as: "There are many dangerous things," "I am hurt because I do not pay attention," "I have to avoid dangerous things and also my mother being mad at me." Then, this network is related to another network with opposite functions (i.e., "things that do not hurt"). For instance, Mario begins to approach objects with physical or arbitrary opposite functions in his history, such as feathers, and things with red color because he has learned that red is opposite to blue. Soon after, he refuses to use a mechanic escalator for the first time and is uncomfortable when any blue object is in his way. He also insists on crossing the street to avoid a hardware store. In all cases, "he is afraid to be hurt." His teacher and family, especially his mother, do not understand these behaviors. His mother says, "I'm tired of your inattentive and stupid actions," while his teacher tells Mario and the family that perhaps he has a brain problem. The point is that Mario has derived punishing functions based on very arbitrary relations. For instance, he was told that hardware stores have all kinds of metal things, and he knows that knives are made of metal. He also has read the written signal "Pay attention: Danger!" close to the mechanic escalators, and these words were related in Mario's history in coordination with being hurt. Finally, the deictic I-other repertoire permits Mario to derive more content about himself and the world around him, such as "I am different from the others," "My brain is different," "My mother does not want me close to her," or "I do not want to use escalators and prefer elevators."

At this point, Mario's fear of being hurt has been expanded based on stimulus generalization and symbolic generalization through relations of coordination, opposition, causality, deictic, and hierarchical framing. The result is a network of words, emotions, and actions coordinated under the function of avoidance (i.e., the fear of being hurt, the fear to dangerous things related to many actions named as "doing stupid things," and related to not taking care, disturbing others, and causing his mother to be mad at him. All the "stupid reactions" he does have been related to "problems in the brain." At the same time, an additional network is formed with thoughts, emotions, and actions based on opposite functions to danger and being hurt. In Mario's words, 
"being secure, escaping danger." Further, more interactions can extend these relational functional operants by deriving more thoughts and actions that will acquire controlling functions. For instance, when his uncle says he needs to pay attention to learn biking, this becomes an aversive action. Therefore, he refuses to learn it because he derives that biking is as dangerous as entering hardware stores and mechanic escalators. Given the derived aversive and avoidance function of biking, Mario stays home and contacts the consequences of not being hurt, which is the opportunity to derive more self-content with aversive and avoidance functions (e.g., relating staying at home with the stable feeling of being secure and perhaps pleasing his mother). This is because his mother sometimes takes care of him even though, at other times, she rejects him without providing clear signals for Mario to predict her reactions. This leaves him without cues to what would be better for him to do to impact the whole components of the networks. As we will explain later, deriving these thoughts and emotions and responding in coordination with the avoidance function they bring is coherent for Mario.

The story continues as Mario becomes more fluent in deictic framing and, thus, can elaborate new self-content by comparing himself to others (deictic I-other) and himself in different times/places (I-now, I-then; I-here, I-there) in conjunction with hierarchical framing where some elements belong to him, and others belong to someone else. For instance, Mario is becoming a fan of the Barcelona soccer team, as his uncle is (both are members in something that integrates them). Because this team is opposite to Real Madrid, Mario jumps with joy when Barcelona beats Real Madrid while shouting "We are winning," as he derives that his uncle and all the fans of Barcelona will be jumping. At the same time, he derives that his brother, who supports Real Madrid, will be angry.

Moreover, when watching a film in the school, he hears that "not taking any risk is a sign of cowardice" and "coward people do not have fun." So, Mario derives that "he will not have fun," which makes him sad. Moreover, while watching other children having fun, Mario derives that they are brave, but he is different and alone. He wants to play with other children, but in one of the few attempts to play soccer, the coach puts him aside for not doing well, and he then escapes running away and crying. He derives that he is a coward and has no friends. So, he stays home alone to avoid being hurt. Even more, Mario's mother frequently asks Mario to be out of sight to avoid being mad at him; she tells him to behave like others, especially as his brother. So, Mario derives that he is silly instead of intelligent as the others are and that he is a problem to his mother. This increases his feeling of being different and not capable of behaving like others.

Due to his fluency in temporal deictic and causality framing, Mario worries about the consequences of not having friends and being alone. Also, he thinks about being brave, being more like others, and behaving like others. So, Mario derives more self-content with aversive functions and is controlled by their avoidant functions that preclude contact with positive reinforcing functions. As Mario frequently engages in avoidance reactions, he is learning to repeat the previous derived content, increasing the strength of the coherence and making it (his self-contents) more rigid or inflexible.

Fortunately, in the middle of these interactions, Mario has had the opportunity to contact contingencies of positive reinforcement mostly linked to activities with his uncle. For instance, he enjoys playing soccer, moving around in the field, tracking animals (especially birds), and playing and creating stories about animals. He also enjoys building different things with lego pieces. He often talks about enjoying these things and says that it is especially true in his uncle's company because he makes sure that nothing hurts him.

In conclusion, all these scenarios have been the occasions for Mario's framing in coordination, distinction, causality, comparison, opposition, conditionality, deictic, and hierarchical to derive thoughts and feelings (that is, more derived contents that are attached to the already derived and now repeated), and to respond in coordination with the functions coming along with the derived behavior. Thus, as a consequence of Mario's daily interactions in the context of his relational history, two networks are on their way. More precisely, two relational functional operants are being built. On the one hand, the one that dominates the scene: a relational functional operant involving many different stimuli, situations with thoughts and emotions showing up and actions, all connected to the function of avoidance (e.g., avoiding being hurt, bothering others and, consequently, approaching opposite functions as the reverse of this network). On the other hand, there is a weak relational functional operant that is being 
built based on all behaviors mostly under positive reinforcement (e.g., the things he enjoys and that he is beginning to be identified with). However, the latter is not built only under appetitive functions of these actions but is also attached to avoiding social and physical risks with the presence of his uncle. That is, the avoidance network, as a relational functional operant, is dominating Mario's daily interactions and is building a trap that blocks other sources of functional control.

The behavioral units forming these relational functional classes are built of multiple relational framings with conditional and, mostly, deictic and hierarchical responding at the core; that is, all relational behavior should be in place. Importantly, here is where hierarchical framing is involved in more sophisticated behaviors, like the formation of abstract sources of motivations along with the functional establishment of ME and the functional organization of the contents connected to I. And yet, one more application of hierarchical framing sets the scene for responding effectively in the presence of whatever private behavior is fleeting in the moment of any circumstance. Let's explore these hierarchical applications, a behavior that would help Mario not being trapped in his own behavior.

\subsubsection{Hierarchical framing in the establishment of abstract motivations as overarching positive relational reinforcers.}

Technically speaking, hierarchical functional classes are built from bottom up, based on common functions from specific actions. These actions at the bottom are arbitrarily connected to a more general and abstract function (going upward), which could also be connected to an even more abstract function. The most abstract function will control the whole network, being this characteristic that defines the hierarchical function. That is, the hierarchical function will be the context for integrating elements with different functions, with all of them acquiring the top hierarchical function while keeping the differential functions. This way, the individual history of interactions selecting the overarching/hierarchical functions will dominate responding even in the presence of other functions. On the one hand, when this is applied to your behavior, the overarching/hierarchical function (e.g., an avoidance function) will control the functions of the different behaviors (e.g., thoughts, emotions, and actions) integrated into the hierarchical network (i.e., it should transform the functional properties of all these elements). On the other hand, the hierarchical functions should set the stage for additional derivations of more elements (e.g., more behavior) under their control. Let's illustrate this with the case of Mario.

Mario's interactions, primarily with his uncle, have been opportunities for learning behaviors under positive reinforcement that ultimately will become hierarchical networks of private and public behaviors. Let's unpack how this could happen. Mario enjoys playing soccer and also moving around in the field to learn about animals, especially birds. $\mathrm{He}$ also enjoys building bridges, villages, and castles with lego pieces, playing with the lego-characters, and inventing stories with numbers. Even though these activities started independently, they will eventually be connected and will extend and amplify their functions beyond the early contingencies maintaining them. For instance, in the interactions with his uncle, a science teacher, Mario has learned to observe the characteristics of birds and other animals. He has also been told that he is becoming an explorer because he asks many questions to know how things work. While Mario is playing with lego pieces and making bridges, villages, and castles, his uncle has told him that he is very good at building and inventing things. His uncle also tells him that being an explorer and inventing things to resolve problems is what engineers do. This dialogue leads Mario to derive that he is like an engineer because he enjoys knowing about animals and building bridges and castles. Thus, "being like an engineer" becomes a hierarchical or overarching function that will control the functions of other behaviors (e.g., playing with lego pieces and observing birds) and will be the context for deriving more elements under its control. For instance, when Mario is told that engineers use math and the English language to resolve problems, his interest in math and English classes increases.

Let's now see an example of how the hierarchical function of "being like an engineer" can also be the context for the derivation of aversive functions. Imagine that Mario's mother does not understand his interest in being an engineer. Instead, she lets him know that engineers are intelligent and brave and do not say nor do stupid things, as he does. One thing that makes the situation even harder for Mario is his teacher describing him as becoming "more impulsive" than before in math and English classes. The teacher tells Mario and his mother that 
he does this to claim attention and that he gets angry and runs away when making mistakes. At the end of the day, Mario feels sad because he will not be an engineer, and thinks he is a coward and cannot do many things. Unfortunately, these interactions actualize previous derived emotions and thoughts, which become the context for deriving more private events with aversive and avoidance functions. The main point is that these newly derived thoughts and emotions become the context for reducing Mario's interest in the activities he used to enjoy because the appetitive network is becoming connected to the avoidance network.

In summary, the hierarchical organization of valuing and establishing relational high-order or hierarchical reinforcers transforms the function of the many different actions integrated in the hierarchical network. This is an RFT conceptualization of values that is clinically very useful (e.g., Dahl, Lundgren, Plumb, \& Stewart, 2009; Luciano, Valdivia-Salas, \& Ruiz, 2012). The experimental analysis of the hierarchical transformation of functions is still on its way, though. It has been partly documented (e.g., bottom-up transformations of functions; Gil, Luciano, Ruiz, \& Valdivia-Salas, 2012, 2014), although the complete picture (bottom-up and topdown) is still in its first steps (Callejón, 2020; Villarroel et al., 2021). Despite the need for further basic manipulations to demonstrate the hierarchical formation of values, a good number of studies in the last fifteen years have shown the conditions under which the domination of a certain function over another is established. We will come back to these studies in the last section of the chapter. Before that, let's look at some more implications of hierarchical framing that establish additional and more sophisticated self-behavior.

\subsubsection{Hierarchical framing in the abstraction of I/ME and the subsequent derivation of more self- contents.}

Whatever behavior a person is doing, there is a constant experience of ME, the one doing whatever is done. However, differentiating I as the context that is the doer as different from the thing done results from an arbitrary -socially mediated- learning process. As stated earlier, this is not an automatic process, but it depends on specific interactions with others, where deictic and hierarchical framing is learned.

From the very beginning of our interactions with others, the words I or ME are incorporated in hundreds of behaviors, all with their respective contingencies. However, these experiences are not sufficient conditions for discriminating or abstracting I from the behaviors done. The others' behavior, in particular ways, is needed to establish the arbitrary differentiation of the agent and the actions done (perhaps, we should remember that we learn the socalled parts as arbitrary divisions because of convenience). That is, the child learns to abstract the differentiation. For instance, from Ieatbanana, Ieatapple, Idrinkwater, Idrinkmilk, Idrinkwater, Iwantplay, Iwantsleep to Ieat banana, Idrink apple, Iwant play, and lastly, to I eat, I want, I drink, etcetera. This abstraction process leads to deriving the experience of I as the only context across all situations. Of course, all this occurs in the context of equivalent examples regarding the OTHER or YOU (Hayes, 1984; Kohlenberg \& Tsai, 1991).

In other words, discriminating the difference between the thing eaten and eating, or the thing being drunk and drinking, and many others, as well as differentiating between the agent and the actions done; that is, the abstraction of I/ME through eating, drinking, feeling, seeing, hearing all are the result of a learning process. This learning requires specific contextual cues provided by others. Questions such as what are you doing, who is doing it, who is doing that now, and there, and then, etcetera, with clear and consistent contingencies are needed so that the child's responses become established in a consistent I across multiple opportunities. The context of the deictic I, in contrast to YOU, with the different combinations of I-YOU and HERE-THERE and NOW-THEN are essential interactions to establish the hierarchical context of an I separated from actions in time and place. The deictic I establishes the conditions for the hierarchical abstraction of I/ME as a "locus" through many different responses in different times and places (see Barnes-Holmes et al., 2001; Hayes, 1984; Kohlenberg \& Tsai, 1991; Luciano, Valdivia-Salas, Cabello, \& Hernández, 2009; Skinner, 1945, 1953). Importantly, as Kohlenberg and Tsai (1991) suggested, when this discrimination process is lacking, the I that emerges is unstable and does not differentiate one's behavior and experience from the others'.

Deictic framing across many exemplars generates the context for a hierarchical I as a basic experience. That is, responding to the question "who is doing X?", with "I am." "It is ME who is doing X." And when doing $Z$, it is ME who is doing $Z$. When 
feeling A, it is ME who is feeling, who is choosing, and so on. That way, ME/I is abstracted as a common function in all these instances. As a child becomes more fluent in multiple deictic framing, using the word "I" (or any other stimuli that should be used for the same purpose) leads to the derivation of a myriad of I-behaviors that were not connected previously but now become so. Accordingly, a hierarchical network is formed with functions coming from all the behaviors, including the initially derived behaviors. For instance, Mario's interactions with the questions of others establish the conditions for him to derive that "it is me who is not playing," "who has been beaten," "who is feeling alone," "who is bothering my mom," and so on. The derived relational behavior coming from these single deictic behaviors conjoint into a higher categorization, ME/I, summarizing aversive and avoidance functions of single behaviors, as a deep feeling of being different, rejected, and alone.

Moreover, the different examples for avoiding being hurt to avoid risks, as well as discriminating the successful common avoidance effects across the different behaviors is the context for deriving the functions of being a "safe person," as a higher-order category of "not taking risks" and with the emotional functions coming from all the avoidance behaviors. Importantly, having learned that "people who do not take risks are losers," Mario derives a more abstract thought and emotion of "me, a loser" that guides the derivation of additional self-behavior and extends the avoidance network, which strengthens its coherence. For instance, Mario rejects a school trip because he is told that it is necessary to be careful and pay attention to the teachers during the trip. Then, he derives that "the trip might be dangerous and to be a safe person you should not take a risk!" increasing the strength of the coherence of his avoidance pattern. Moreover, because no alternative conditions are given to Mario but those that maintain the avoidance pattern, "the qualification as a safe person" is extended throughout the coming years. For instance, he might be invited to a party in his adolescent years, but he refuses in order to avoid the risk. Even later on, he might refuse a job because he feels he cannot do things by himself and needs help and support from others. Given these conditions and Mario's fluency in relational responding in deictic, causality and comparison, he will derive more general thoughts with the corresponding function. For instance, "I will never be normal as the others," "Nobody wants to be with me,
I need to be out of view," and so on. To the extent that relational responding is reinforced, the person will develop greater fluency in deriving relations according to causality and comparison based on deictic. For instance, when Mario realizes that things are inconsistent with what he thought should be (i.e., when confronting problems), his primer reaction is to derive reasons based on his fluency in framing stimuli in causality and comparison based on deictic framings I-Other and I-Here, I-There. This way, Mario asks and responds to himself why things happened and compares himself at different times or with others. Consequently, many thoughts are derived as reactions to the problems, thus, becoming components of the functional relational operant as additional multiple exemplars. Needless to say, the relational avoidance operant is augmented with every single unit and it strengthens according to the coherence established in Mario's life.

At the very end, Mario feels deeply different from others, a loser, unable to do many things, and living in an automatic way. Because he typically responds according to the avoidance functions of private events, Mario has multiplied that type of reaction to his world inside when contacting the world outside. Furthermore, he is having many limitations in his life and deriving more fears and more suffering. In his middle twenties, Mario expresses that he feels like "a mouse escaping to the holes" most of the time. Generally speaking, Mario is deriving a metaphor as relating two networks. On the one side is the network with the causal relation established in Mario's experience with mice (e.g., "the mouse running away to the hole and escaping from the cat"). On the other side, his network of "running away from others' view and keep hidden to avoid being hurt and rejected." Given such a context, he derives more relations with the respective aversive functions as "he sees his life as a desert with nobody loving him, unable to do many things and not recognizing frequently who he is." Moreover, in such a context, additional thoughts are derived as "no solution, nothing to be done, not being really alive, and not standing things being that way forever."

Importantly, the self-contents become frequently organized in hierarchical networks. The different groups of networks at the bottom gather into a more abstract categories with higher aversive or appetive functions (depending of the personal history) that finally derive at yet another more abstract level, all under the rubric of ME. That is, some private events 
might be concrete (e.g., "I failed the exam," "I have not gone to the party." "I can not reach their attention"), with the specific function they bring according to a particular learning history, and become established at the bottom level of the hierarchy. Other verbal formulations are more abstract and connect the more concrete ones according to the particular person`s history, which lastly are connected to the continuous experience of ME (e.g., "I do not do anything right," "I am a disaster,". Moreover, they can go further up with increasing abstraction, and the higher up in the hierarchy, the more dominant/inclusive the function is, again, according to the person`s history (e.g., "Nobody loves ne," "I feel alone and empty") . Importantly, the functions integrated at all levels become connected to the continuous experience of ME with further derived responding (e.g., "What kind of person am I?," "I hate myself").

Recent research (Gil-Luciano et al., 2019) has shown the frequent hierarchical organization of negative thoughts in most participants. They reported a higher aversive value in those thoughts that they have established at the top of the hierarchy compared to those at other levels. Beyond the clinical observations, this is congruent with the scarce available data on the hierarchical transformation of function. As indicated in the previous paragraph, research has shown the transformation of functions from the bottom to the top level of hierarchical networks (Gil et al, 2012, 2014). Furthermore, if a new function is given to the stimulus at the top of the hierarchy, it will be expanded to all the network members (top-down) (Callejón, 2020, and Villarroel, Luciano, \& Ruiz-Sánchez, 2021). This means that thoughts about oneself established during life, each with its specific functions, when organized in a hierarchical network, those at the top affect the meaning of all the hierarchy members.

Now, we will describe yet another application of hierarchical framing, perhaps the most complex one. As indicated in other sections of this chapter, we always respond to the stimulus functions in the present and we do so in the context of our personal history. Accordingly, when thoughts and emotions are derived, we may learn to choose the direction of our response and what specific response to do, but we simply cannot choose not to respond. We give the last portion of the second part of the chapter to describe two patterns of responding to your behavior, with opposed functional focus, and, accordingly, different effects for the person and the whole community of individuals that establish what is meaningful in the respective culture.

\subsubsection{Two types of responding to one`s own behavior as two relational functional operants}

As described previously, human beings are constantly visited by emotions and thoughts coming along in particular moments because of their personal history. These behaviors are brought with motivational and discriminative functions. The first and easiest way is to respond according to these functions, which generates reinforcing consequences accordingly that sooner or later will be transformed into suffering. This behavioral pattern is known as psychological inflexibility and is the relational operant dominating Mario's repertoire. The other more complex way of responding to one's own behavior, named psychological flexibility, is characterized by responding to the your behavior under the control of meaningful personal motivations that should be established as a hierarchical function integrating any other present functions. Consequently, at least two functions need to be present, one that will become dominant (e.g., something to become meaningful for the person) over the other (e.g., fear, doubts, etc.). Because of responding to one function over other different functions, the former becomes an overarching function connected to I. This is the core of the repertoire of framing one`s own behavior hierarchically. As stated previously, this has to be specifically learned.

\subsubsection{Psychological Inflexibility as a pattern of responding in coordination with the one's own behavior. The pattern of psychological inflexibility is defined as a relational operant established by multiple exemplars (MET) of responding in}

coordination with the function of one's own behavior (Törneke et al., 2016). The most frequent function controlling this operant is responding to the avoidance functions that private events bring, as established in opposition to doing things in a meaningful direction. Although exemplars of this type of responding typically pay off in a short time in the context of avoidance, it does not consider what matters in each moment. Thus, this operant does not work in the long term because the result of the whole package of MET prevents contact with personally relevant contingencies. Consequently, when this 
operant dominates the person's repertory (e.g., it is the unique or most frequent operant), suffering and limitations in the own life are on the stage.

Let's think of Mario's history, where some appetitive functions that matter to him have been contextualized in opposition with specific private events. Consequently, when signals of fear, hurt, disapproval surface, avoidance function will dominate the scene. Then Mario displays different actions established in his inflexible operant along with his life (e.g., crying, running away, staying alone, complaining, and thinking about how to get away from all this). These actions are frequently effective in the context of avoidance but not in the context of the functions that, at the very end, matter to him. Accordingly, he is trapped responding in coordination with avoidance functions based on the instructional control of rules inviting him to fight against certain emotions and thoughts. In other words, Mario displays a pattern of responding where avoidance functions dominate in a never-ending cycle of avoidance that prevents contact with the contingencies that seem significant for Mario.

According to the conceptual and empirical research conducted, let's summarize the relational characteristics of this inflexible generalized functional class as an ineffective relational functional operant.

First, as all functional classes, relational functional classes are operants, established at the individual level and through multiple exemplars training (MET). Consequently, the repertoire of responding in coordination with one`s own behavior is established in different ways across different persons, but all having that specific function.

Second, as an operant, topographical variability in the MET components for each person is key to building this repertoire. However, all behaviors are established in the context of a common function: responding in coordination to the avoidance function of private events. Then, the MET along the personal history shows variability in its components. That is,

(a) Variability in the physical circumstance or stimuli that bring different functions as variability in private experiences: the thoughts, feelings, and emotions are related in particular ways because of the specific history of relating (e.g., the rules established in the person's life).

(b) Variability in the different ways of reacting to the functions of such private events: different reactions that are under a common function, say avoidance, based on the rules established in the person`s life. For instance, avoidance behavior such as thinking over and over, consuming drugs, alcohol, crying, complaining, doing physical exercise, oversleeping, etcetera. In turn, the consequences linked to the avoidance reactions are mostly negative reinforcement (the discomfort, fear, pain are somehow alleviated) and all under the coherent reinforcing function of relational responding.

(c) Importantly, in the vast variability of exemplars controlled by the common function of avoidance, there is typically a way of reacting to problematic private events. This is engaging in a cycle of "thinking and thinking" that does not solve the problems but occasions a motivational context for additional avoidance actions. This way of thinking, frequently known as rumination or repetitive negative thinking (Ehring \& Watkins, 2008), is a pervasive, primer way of reaction that occurs according to the coherence of the relational history (Ruiz, RiañoHernández, Suárez-Falcón, \& Luciano, 2016). As well, the resulting thoughts and emotions of this relational activity shows variability that depends of the particular fluency in relational framing when problematic private events are present. For instance, it might be given reasons as a function of comparing oneself in other times and places; or with other persons, or based on asking for/answering on causality, etcetera.

(d) Variability in motivation, that is, in the quality and the meaning of acting. These motivative functions, frequently implicit or not well discriminated by the client, are not contacted because actions under its control are blocked by the domination of avoidance control based on relating aversive private events as opposed to valued actions. Consequently, the avoidance operant imprisons the person his own behavior.

Third, as coherence is the hierarchical function that characterizes the history of relational responding, Then, all the components of the operant, including the consequences of responding, are contextualized in the relational history that is brought to bear in any situation. This is why responding under the control of avoidance function makes sense even when it generates suffering in the context of higher-order or meaningful motivations (that are seen as opposed to the functions controlling avoidance). Once again, the reinforcing function of the coherence of relational history makes sense, perpetuating the pattern of responding. 
Fourth, as an operant, it is defined functionally and has been found transversal throughout most of the formally distinguished syndromes in mainstream psychopathology. Furthermore, it has been found transversal in many other areas where the human condition generates some level of suffering or discomfort in pursuing life. Finally, the research evidence regarding these behavioral patterns is extensive in many respects (the reader should find some examples in the final part of the chapter).

\subsubsection{Psychological Flexibility as a pattern of responding hierarchically to one's own behavior.}

In the relational definition provided in Törneke et al. (2016), this pattern is seen as a functional class of responding characterized by framing one's own behavior in hierarchy with the deictic I. Put briefly, psychological flexibility is a relational functional operant of framing one`s own behavior

\section{hierarchically.}

What is needed for such a repertoire to be established, at whatever age, is a MET of specific responding for learning to not respond directly to the functions showing up, being appetitive or aversive, and being aware of noticing them and responding in the inclusive context of another function, typically, a meaningful, higher-order or abstract function based on positive reinforcement. Learning such an operant involves a complex process of becoming conscious of ME (deictic I), that is, of who is noticing the functions fleeting as well as of learning to make choices and responding under the control of that function that we would want to control our behavior. This way, when MET of this type of responding is in place, one function acquires a dominating hierarchical function over other functions. This is the case of Maria, who is having similar interactions with her family as Mario does in early childhood. However, she was moved to live with another family who fortunately provided her with a motivational context based on positive reinforcement. In this new context, she learned to be willing to contact her avoidance rules and still acted according to those other functions. That way, Maria was able to build a consistent and transversal ME through effective ways of interacting with her private world. Rarely, we learn to fly with the winds of pain -whatever formwithout a systematic learning process that has promoted responding focused on a meaningful function and integrating -instead of escaping from- the pain in the way of acting under meaningful function.

Even Mario could have been quite a different person if he had been exposed to another history of interactions with others. A learning history that could have established an inclusive relational pattern of responding to his own behavior, as in the case of Maria. All in all, Mario is in a position to learn an effective relational operant if given the contextual conditions for learning a relational operant that will allow him to experience the contingencies available when choosing consciously to respond under the functions that might be developed as personal meaning. Said another way, if Mario is given conditions for effective interaction with his own behavior, then a hierarchical network -integrating different functions and actions under one dominatingwill be established. That is, he can learn an effective repertoire consisting of integrating whatever functions show up, according to the networks established in his history, while having space to respond under the influence of the function most meaningful to him. That way, Mario will learn the flexibility repertoire, perhaps the most complex of the legacy of our ancestors via the culture where we are raised.

Before going there, let's notice the characteristics of psychological flexibility as seen from the perspective of a relational operant as hierarchically responding to one`s own behavior. Many key characteristics of the context, indicated previously regarding Maria`s history, could remain the same except that the relational and functional contexts controlling the MET are radically different and, consequently, a different repertoire is established.

This repertoire is built through a deictic/hierarchical MET that connects the diversity of thoughts and emotions, and the functions they bring, to a diversity of actions all under the control of an inclusive function of other present functions to allow a better adjustment to the personal meaning.

First, the type of deictic/hierarchical MET is defined by hierarchically responding to private events, of course, in the context of the deictic I. Each exemplar is a chosen act under the function connected to the hierarchical ME, noticing the act of inclusion/integration of the thoughts and emotions that come in the way. This means that each chosen act is done in the deictic I-Here and my thoughts/emotions in the deictic I-There, generating a 
space between these events and what really matters to me.

Second, variability in the MET is key in building this operant as any other one. It will be variability in the content or type of private events contextually depending on the present and historical contexts; variability in the personal meaning, although finally all connected to the experience of ME; variability in the actions chosen and, consequently, variability in the formal consequences that finally will be transformed according to the personal meaning as the higher or hierarchical common function across multiple exemplars.

Third, as previously indicated, coherence is the reinforcing function for responding according to the relational history that is brought to bear in any situation and is transformed accordingly. In this case, in the common context of the actions chosen under the hierarchical function.

Fourth, the relational operant of responding hierarchically to the own behavior is a behavioral pattern identified in many areas as an effective repertoire of psychological flexibility: from clinical to sport, business, and policy; from adults to adolescents and children. That is, it is transversal_and seems to be the most effective way to interact with our condition as verbal humans.

When that type of MET has not been provided to a sufficient degree in the personal history, as in Mario's interactions, there is still a way to go, a repertoire to be learned. This is the process of therapy, a process of building a repertoire of flexibility in the context of the pervasive coherence where the dominant functions controlling the client's behavior are ruining the client's life. In basic terms, building a relational operant of responding hierarchically to one's own behaviors on the grounds of the pervasive relational operant of responding in coordination.

\section{Clinical strategies as MET to build a pattern of hierarchical responding to one's own behavior}

Törneke et al. (2016) described three core strategies to build the pattern of psychological flexibility as responding to the ongoing private events in hierarchy with the deictic I: (1) Helping clients experientially contact their history of inflexible patterns, (2) Helping clients build the ability to frame their private events in hierarchy with the deictic "I," and (3) Helping clients clarify and amplify hierarchical positive motivations and choosing actions linked to them. The three strategies constitute a whole package, and, consequently, all their movements are multiple exemplars to help the clients display the hierarchical response to their behavior. The following paragraphs focus, firstly, on the functional analysis as the basic strategy and, secondly, on different aspects of the whole process that conjoint to the final common pathway of hierarchical responding. Thus, this is a more technically precise way of describing what was described as three strategies in our earlier publications.

\subsection{Functional analysis as the basic strategy}

Functional analysis is not a specific recipe but a functional perspective applied in different ways and for different aims. It can be applied to clinical work, basic or applied experiments, teaching, and many other types of action. From the very beginning, the $\mathrm{ABC}$ unit has been successful in altering or building operants: (A) the historical and present antecedents (motivational and discriminative functions in a particular moment), (B) the response to such functions, and (C) the consequences (as positive or negative contingencies of reinforcement). This functional unit of analysis is sufficiently flexible to be also used also when the ABC is applied to the typical relational behavior of verbal human organisms. A threefold focus is needed to clarify these characteristics.

First, any present behavior results from a personal history and is mostly exemplified as an operant established, then, through a history of MET. When conducting a functional analysis, we look for the operant by examining the motivational and discriminative functions of antecedents, the different responses under such functions, and the common consequences that follow across different but functionally equivalent situations.

Second, when a verbal repertoire is in place, the functional analysis becomes a relational functional analysis (Luciano et al., 2020; Törneke, 2021). That is, the $\mathrm{ABC}$ now involves functions established through relational responding, but functions nevertheless. So, we analyze relational motivational and discriminative functions of stimuli, the behavior that occurs under such functions, and its consequences, aware that these consequential functions are also established by relational responding. All this occurs in coherence with a particular relational history. Therefore, each 
examined behavioral unit (flexible or inflexible) will exemplify typical thoughts, instructions, and emotions of the client, their motivational and discriminative functions, the multiple behaviors that follow, and the consequences in the short and also in the long term.

Third, when doing a functional analysis as part of therapy, the goal is to generate the conditions for the clients to discriminate and experience their way of interacting (B) with their private events as the functional echoes of their relational personal history (A), and the overall effects of this responding (C). To say the same thing in a different way: to help the clients become aware of their historical and present baggage and, given that, be confronted with, "and then what?" In other words, the therapist helps the client to become aware of the historical and present sources of behavioral control in a way that opens the door to behavioral change.

One important thing to stress is that fostering a flexible repertoire is not just talking about behavior but generating the conditions for relevant functions of private events to be present, helping the client to notice them, and choosing the best response in such conditions.

\subsection{Analyzing interactions in the moment}

A preliminary point is that the function of the therapist's behavior is not in the therapist's behavior per se, but the function is in the meaning for the client according to the client's relational history. Thus, the flexible relational functional class will be promoted through multiple exemplars, which must be understood in that context. This point connects directly to the earlier formulation of clinical behavior analysis (Dougher \& Hayes, 2000; Ferster, 1974, Kohlenberg \& Tsai, 1991). The critical point for the therapists is to be aware of the effects their own behavior produces in the interaction and rely on that analysis, rather than on rigid rules about what might be the result of any action from their side. That is, the meaning of something is not in the physical properties of the stimulus, in the content of a particular sentence or a particular posture, but in the client's history. All this occurs, from the beginning to the end, through the functions that the client's behavior generates in the therapist's behavior and vice versa. So, the therapists need to look for many different ways to adapt their behavior to the client's repertoire to support the client's learning process in moving from responding in coordination to their own responding to responding hierarchically in the same context.

For instance, even as the therapist might start the first session of therapy by asking permission to question about sensitive issues in the clients life and encouraging them to stop her whenever they want to, the therapist should notice the response of the client to this basic therapist behavior. The therapist's intention, of course, is to establish a shared context between therapist-client as a starting point for communication. The critical point here is that even such basic phrases, and other signals that the therapist might show, have to be functionally analyzed in the context of the client's responding. Accordingly, the therapist's continuous task is to explore how the client's behavior changes in the session.

All strategies used by the therapist to support the clients in their change process are played out in the interaction between the client's and the therapist's relational behaviors. This means that all types of relational responding will occur, as they probably are in any kind of verbal interaction. To accomplish the MET needed to increase psychological flexibility, we will emphasize hierarchical responding as the most versatile and central repertoire to train. In the following section, we will thus describe the work centered around this training, at the same time as we will present some different aspects in the process based on Torneke et al. (2016) and Luciano et al. (2020).

\subsection{Helping clients to experience the repertoire of responding in coordination to one's own behaviors and moving to responding hierarchically to them.}

The therapist's behavior is focused on generating a context of interaction that increases the probability that the clients will become aware of their inflexible behavioral pattern and how it relates to what is meaningful to them. In each of the processes of discriminating one's own behavior on the one hand and what is meaningful to oneself on the other, hierarchical framing of your own behavior is key. Consequently, the therapist tries to have the interaction move between the components of the inflexible repertoire to meaningful motivation (and vice versa) and constantly focusing the training of hierarchical framing of one`s own responding as focus. The different clinical interactions used to accomplish this can, in a simple way, be summarized as: "What is the problem, what has been done to 
resolve it," and "what are the effects regarding who you want to be and to accomplish?" Questions and answers, exercises, and metaphors co-created with the client are used in this training.

As we continue to describe some clinical strategies to be used by the therapist, we want to caution the reader that the following paragraphs do not pretend to be a list of items to follow in a strict sense but only a guide to support the therapists in their efforts to make the interaction a learning experience for the client, so that new, flexible behavior emerges.

\section{a. Helping the clients to (1) differentiate the private events, including their rules, as motivational and discriminative antecedents under which they respond in coordination, so that they start to (2) differentiate hierarchically the one who is noticing and who is responding.}

There are many types of interactions exemplifying this strategy. For instance, the therapist reacts to our client Paul`s different behaviors as follow:

"You say that when you are with people, as you are now, here, with me, you feel anxious... How do you notice it, where in your body do you feel your anxiety...? Are there other situations when you notice the visit of this feeling of anxiety?

"What thoughts come to you right now, feeling that anxiety in your chest? Alternatively, "You say that you have the thought of being a disaster. What other thoughts are coming with disaster? What is your body feeling when these thoughts visit you?"

"There are many ways of feeling anxiety. In your case, can you tell where you notice the feeling of anxiety? Can you put your hand at the place in your body where you feel the anxiety? How is that? What kind of anxiety is it? Let's imagine that anxiety has a form and a color. What might it be? Let's think that you can touch it and you feel its temperature, how is that? Can you leave the anxiety in your chest for a while and put your attention somewhere else in your body? For example, can you move your attention to your right leg? Now you can move your attention back to your chest and notice you are back there... Can you, once again, move your attention to any part of your body of your own choice? Do you notice that you can move your attention? (the client says yes). Let's notice that experience and stay with it for a moment... Now, go back to notice the anxiety in your chest... Notice that you are noticing what you are doing... Can you bring a picture of you when you are at home with that feeling in the chest? Any other picture of you when you are at work? Can you bring some more pictures of you when you were an adolescent in a moment at school? Whatever picture is OK... Can you move your attention from one picture to the other? What about imagining yourself as a lighthouse that moves attention to different things occurring to you?"

If our client, Paul, has followed this interaction and there are signs that he is discriminating himself as the observer of his own responding, the therapist might continue with asking the client to imagine or draw out this experience on a piece of paper and shares, pointing to different perceptions noticed, the one noticing and the lighthouse shifting the direction of the light.

"Although there are differences between us as humans, we all react to what we feel and think... In your case, how do you react when your anxiety, sadness, loneliness, and worries about the future talk to you? You say that you do not want to feel that way, that you don't want to have the thoughts you have... You say that you react in different ways to stop thinking and feeling these ways... When you feel..., you do..., to avoid feeling worse... When you feel..., you do..., And who is noticing all? Who is noticing the feelings, the thoughts, what you do, who is noticing? (The client answers: "It is me, I guess..."). Yes, so you are more than all the things you can notice with yourself in that sense. You are also the one noticing them; might you also realize being the noticer.?"

\section{b. Helping to potentiate the hierarchical experience of $I / M E$ as the transversal functional context of my behavior.}

Sooner or later, the therapist should help the client derive the hierarchical experience of ME as the permanent and hierarchical context of his own behavior. This is not only tacting the ME but also to ensure that a common experience throughout all different behaviors, of what I felt, what I am feeling now, and might be feeling if, of what I have done, am doing, and might do, of what I am thinking about the future, about the past, about my family, my country... Over all this, I am experiencing or noticing the context of Me noticing and Me reacting to this unique experience...

"Let's put it this way, on a side, it is you feeling anxiety, and then you also feeling sadness, it is also you feeling alone, and you feeling rejected... It is you thinking that your mom is..., and it is you seeing the 
past..., and you seeing what it would be like if you were not feeling alone or rejected..., it is you in all these situations. On another side, it is you as the observer of one aspect, you moving the attention to some other, then to one more, as if being a lighthouse moving the light from one place to another. Even more, there is other orbit besides observing your thoughts and emotions. It is the orbit of you reacting to any of them, in one case, you did..., in another, you did..., now you are with me talking about this..., What do you see here? What do you notice?

The therapist moves to help the client, Paul, derive the hierarchical I across many of his own behaviors to foster the hierarchical organization of his behaviors as different elements of his problematic strategy or repertoire, the one of responding in coordination. The therapist is using many different cues to that aim. In all cases, the therapist tries to train the experience of I-HERE and behavior as ITHERE, and finally hierarchical framing in different domains.

\section{c. Helping to give a common, hierarchical name to different private events.}

One more example of hierarchical responding has the aim of helping the client to derive a common name for the multiple private events that have, through the personal history, acquired problematic functions. Putting the different events together is useful to support the discriminative process. Even if not all have the same weight, they belong together as they share a common function, typically avoidance. For example, our client, Paul, might spontaneously name his thoughts and emotions as "alarms in the mind," or as "devils and angels in my head," or someone might describe his urges as "waves and storms in the sea." The therapist should be observant of these kinds of expressions, and when they seem to be helpful, to catch and use them. The therapists might also introduce a metaphor themselves. When Paul talks about worrying thoughts disturbing his sleep, for example, the therapist can say: "Yes, thoughts can be like irritating flies that keep you awake at night. You try to fight them, but they seem to be coming back and coming back." Let's say the client answers this by saying: "Yes, there is a whole bunch of them, one worse than the other. Then the therapist can go on and co-develop the metaphor by, for example, asking the client to specify a couple of the worst "flies," perhaps giving them each particular names.

\section{d. Helping to identify the different ways of responding connected to the same hierarchical function.}

The same strategy of giving a common, perhaps metaphorical, name is used to help discriminate the different behaviors sharing the same function. This can be applied to inflexible and flexible classes of behavior. One important class of behavior to label this way is, of course, the different forms of experiential avoidance. For instance, the therapist might say, "When you have all these flies trouble you and block other things you want to do, what is your reaction? What do you do? In this process, the therapist tries to help the client experience that different types of behavior are going in the same direction and have the same function. Perhaps these different behaviors can all be gathered together under the heading of "trying to strike the flies." In that way, the therapist can also help the client derive the relationships between the "flies" and the rules, they carry with them, leading the client into problematic circles of avoidance, the effort of "trying to strike the flies."

Particularly important is to help the client, Paul, to notice the quick, almost automatic, way he interacts with what turns up for him. These are the "self-evident," assumed "necessities of action" that constitute the motivational and discriminative functions the client tends to frame in coordination rather than hierarchically. These quick responses can be overt behavior but are also often ways of thinking and further elaborating about the problem experienced. The relational functional class of responding in coordination is frequently dominated by particular ways of thinking and thinking about the thoughts and discomfort that worries the client. When this is the case, the therapist should help the client derive the why as the rule instructing these reactions. For instance, the therapist can help Paul identify the relation between the never-ending cascade of thoughts and emotions as, finally, the consequences of his efforts as indicated in the next point.

This way, the client will have the opportunity to contact that many different things they do are ways to escape or avoid aversive thoughts and emotions and that they all constitute the same strategy, providing the same problematic consequences.
e. Helping the client discriminate the causal relation between private events, responding under their control, and the short and long-term effects. 
The therapist can ask questions to help the client discriminate the whole problematic pattern of certain private events, their functions, and the short and long-term consequences of following such instructions or rules. This can be described as the temporal relations of the sequence of events that should be discriminated as explicit causal relations. The therapist should assist the clients in deriving cause-effects relationships across time between what they are looking for and the consequences they are pursuing both, regarding the maintenance and the expansion of the unwanted thoughts and emotions, and regarding the persons they would like to be. These interactions aim, firstly, for the client to contact the aversive functions provoked by responding in coordination as well as by projecting these aversive functions into the future according to the clients experience. Secondly, deriving the aversive functions will hopefully serve as motivation to contact higher reinforcing functions connected to them, that is, things that are of utmost importance to them in the long run. Of course, the process might go in the opposite direction, as when the interactions are first helping the clients to derive seeing themselves behaving in hierarchy with what is meaningful for them and, from such a context, seeing the aversive function of responding in coordination.

The therapist might point to the causal chain of events that the client, Paul, first described as separate events. It can be done by saying, for example, "Let's look at this as if watching a film of you. You say that you are visited by anxiety and emptiness and then you have to do something to get rid of them. You say that you can not pursue your life when those feelings and thoughts are visiting you. Is that correct?

Moreover, I realize, being here with you, that these experiences are very uncomfortable. Furthermore, when these feelings and thoughts are present, then you do different things to stop them, the alarm, the flies (or whatever term is helpful.)" If the client then seems to agree with the formulation (and it is very important for the therapist to evaluate whether that is so), the therapist can continue: "If you were not visited by these thoughts and feeling, would you be doing all these things?" Assuming that the client answers that he would not, then the causal relation is discriminated.

Once again, it might be useful to help the client to formulate a metaphorical name for the I who is responding. It might be the driver, the captain of the boat, the boss, or something else. In addition to strengthening the experience of being capable of choosing what to do, it can help to connect to what is ultimately important to the client. For instance, what type of driver do you want to be: one that leaves the bus under the control to the fliers or the alarms posed by the passengers, or the one who is in charge of the direction of the bus?

Once again, all types of relational behavior are present in this type of clinical dialogue, but hierarchical responding is "the final common pathway" in establishing clinical change.

\section{f. Helping the client to integrate the most aversive private events into the hierarchical ME.}

As indicated in the second part of the chapter, the networks established in the client's life are functionally coherent. They are built by deriving selfcontent and repeating them as well as the rest in the respective network. Any of the self-contents might have a relational discriminative function for more derived content. Helping the clients to experience these networks, as the echoes of their personal history, seems to be a beneficial step in the process of integrating all and reacting to them.

For instance, when Paul is experiencing a problematic thought, the therapist might help him give it a name (e.g., saying, "this seems to be a big one, doesn't it?). Then, the therapist might invite the client to look for other thoughts that might be behind or around, even with most aversive functions. That is, on a side, the client is helped to give a name (perhaps the big thoughts for the most aversive ones, and the little ones for another with less aversive functions). On another side, the client is helped to take a distance from all of them, especially the big ones, and integrate them into himself while moving forward. Typically, experiencing the emotional functions of the most fearful emotions and thoughts might be hard for the client because they are connected in some way to what is meaningful for the client.

Consequently, this might be a key motivational context for helping the client to notice and integrate them and overcome the blocking effect.

\section{g. The history in the present. Connecting private events hierarchically to the client's history.}

Helping the client, Paul, identify causal relations between the most problematic and painful private events, that he is experiencing now, and episodes in the client's history, seems to be an effective step in establishing a well-functioning hierarchical experience of self. This is especially needed when the echoes of history prevent to focus in what matters. In 
such circumstances, the therapist should invite the client to go over them looking for moments, mainly those early in life, when these thoughts emerged to help the client integrate all the emotions involved, and moving forward..

The process of supporting the client in this work is in many ways similar to what has been described previously in this chapter. It typically adds dialogues and perhaps exercises where different key memories from the client's life are explored, and relational cues are provided for our client, Paul, to see himself from the perspective he had at the time of the event described. That perspective is then coordinated with the one experienced in the here and now by questions like: "Right here and now, can you notice that little child at that time? And can you notice that the one who was there and then is also YOU, being here with me?" This can be elaborated in many different ways, for example, by asking Paul to switch perspective, at one moment responding from the perspective of himself within the described event and in the next from the present situation. Moreover, as this is done, relational cues are provided for integrating the different perspectives as "ME."

Sometimes, exposing a client to specific moments like these will cause very strong emotional responding. Therefore, the therapist should follow the client carefully, asking permission to continue or to stop. In the latter case, the therapist might later ask the client to go back and be willing to learn the repertoire of being the observer of the echoes of such episodes, and moving forward under meaningful functions, that is, as instances of hierarchical responding.

\section{h. Helping the clients to experience the impact of the long-term and accumulative consequences of responding in coordination with their own behavior.}

Under a previous heading (e), we discussed helping the clients contact short-term consequences of their present, problematic behavior. We now want to give some extra focus to long-term consequences. As previously indicated, this is not merely discriminating a chain of events but deriving the causality between what they are doing and the consequences in the long term. This should be examined in the context of what might be ultimately meaningful to them.

The task is to help the clients contact multiple examples of short-term consequences and asking the clients to compare those with the effects in the long run. This can be asked in the context of the life they want to live. Are they more or less tired now than at the onset of the problems? Do they experience themself as stronger or weaker? The disturbing thoughts and feelings that they experience, in what way does their present strategy affect those?

Compared to some time back?

The therapist might say: "Imagine yourself in a couple of years, continuing to do what you are doing now? What if you continue to fight the flies! What will your life look like? What about your dreams, your emotional situation, the sense in your body...?"

This work will hopefully open the client to look in new directions. "What would it be like to be the captain of your own boat? In that case, where would you be heading? What kind of action now can take you in that direction?" If the client follows in the interaction, the therapist can ask: "How do you feel when looking at this? How does it sound to you?" Hopefully, the client will respond with curiosity, experiencing this as something different. There might still be barriers, the uncertainty of what this implies. However, a possible way forward is present.

\section{i. Helping to co-create a metaphor for the whole intervention model.}

Using a metaphor that contains all the components we have discussed, from responding in coordination with your own behavior to the alternative of responding hierarchically, has been found clinically and experimentally useful (Peña Vargas et al., submitted; Ruiz et al., 2016; Törneke, 2017). Metaphors can help the clients see the whole picture of their behaviors and open the horizon for a new way to act. For instance, some clients might say that their head is full of alarms or flies, or some others might describe their life as an empty desert or, as our client Paul, his situation as running like a hamster in a wheel for years. The therapist might respond to these remarks by trying to co-create an overarching metaphor to help a client derive the components of both the problematic pattern and a possible new avenue. For instance, in responding to the description of Paul as being like a hamster:

"It seems that you do not like this sense of being like a hamster in a wheel. I see hamsters enjoying the wheel, but what about your experience of feeling as if being a hamster?" In a dialogue based on the client's experience, and through the lens of this metaphor, all aspects of the Paul's situation can be looked at. What occurs inside Paul that makes him jump into the wheel (examining private events and their functions as antecedents)? In what specific ways do he actually 
runs, how is he behaving inside the wheel (what actions are taken)? And what is he obtaining when running in the wheel (asking for short-time consequences linked to the rules followed)? What things are important to the client, outside of the experience of being a hamster (asking for possible meaningful life patterns)? Or in the same direction: "If you could choose not to be a hamster, what type of animal would you like to be?" If Paul, for example, would respond that he would like to be a dolphin, being able to swim and jump in many different directions, then that metaphor can be used to explore the quality of the actions coordinated to being as a dolphin. If Paul should respond that "being a dolphin is being free," then the therapist should explore the possible alternative strategy and the possible consequences of acting with freedom.

\section{j. Helping the clients to potentiate the ability to frame private events in hierarchy.}

We have emphasized the central repertoire of hierarchical framing of your own responding through this section of clinical intervention. Let us now add some directions to promote this behavior when the client does not show fluency in doing it. Like learning other abilities, MET to establish this behavior should include variability in the content but keep common relations. According to Luciano et al. (2011) and Luciano, Ruiz, Gil-Luciano \& MolinaCobos (2021), training hierarchical framing of your own behavior is connected to training deictic framing.

To help the clients to derive the deictic relation I-HERE and their private events as I-THERE, MET might proceed from stimuli with neutral functions (e.g., the thoughts just emerging when looking at the glass, the lamp, or the window) to those stimuli with appetitive and, mostly, with those with aversive/avoidance functions (e.g., the anxiety or the problematic thoughts). Several deictic and hierarchical relational cues should be used to encourage the response of observance and integration of thoughts or memories (e.g., "contemplating the thought as if you were contemplating a painting" or "writing it down on a post-it on the wall or writing on a balloon, if written in lower or uppercase letters," etc.). In the case of emotions, the client might be helped to physicalize them, for instance, as if they were objects in some part of the body, and asking for what kind of object might be, what color if having a color, or size, what temperature? and so on. In all cases, the aim is to promote a kind of distance and, then, of integration of the private events as transitory events that one can observe without jumping into them and being away from what matters. The training would encourage experimenting with the fact that one can observe one thought/emotion here, another one here, another one there, and so on. In this process, the therapist directs Paul`s attention to the behavioral options connected to what might be meaningful for him, which would constitute hierarchical responding to overarching consequences for the person in question.

One additional point in this process is to help the client, Paul, experience the very act of choosing behavior, the awareness of being the person who is doing the chosen act and connecting this experience to other acts and goals. That is, deriving the experience of himself hierarchically as "the I" who makes choices, who acts, who realizes the quality of his acts is the central step in amplifying the relational operant of hierarchical framing of your own behavior. The final derivation promoted in the clarification and amplification of meaningful functions should be realizing that any private event is one aspect of oneself and that one is much more than any such single feeling and thought. What should be promoted is the experience of being a permanent transversal context of observation, having the ability to give space for whatever turns up on the private scene, and of making choices of what direction to take from there.

\section{k. Helping to clarify and amplify the hierarchical function of meaningful directions}

In clinical situations, the experience of appetitive motivation is typically blocked by private events. Therefore, establishing such motivation as hierarchical and helping the client to act under such overarching functions is a critical aim. A key way for the therapist to increase the probability for this to happen is helping the client to frame different actions and behavioral goals hierarchically, so that all of these become connected to what is experienced as ultimately important and meaningful.

This work involves interventions from the bottom of the hierarchy and upwards and interactions moving from the top and downwards (Luciano et al., 2021). To exemplify, imagine a client who has suddenly lost his wife in close connection to also being retired from his work as a teacher. He summarizes his present situation as "the end of his life" and isolates himself from most activities outside his home. At the same time, as the therapist validates 
the client's loss, she might proceed by asking questions like: "In your life together with your wife, what would you say were most important things for you? What carried the deepest meaning, those feelings that were present when you were doing things together?" The same type of questions could be asked about his experience as a teacher. In these questions, the therapist is looking for an overarching motivational function. Once something is made explicit, it can be used to move "downward" in the hierarchy, connecting it to specific acts and possible consequences in the present situation. So, for example, the therapist might continue: "So, this creativity and intimacy that you described from your earlier working situation when you were teaching your worst students, and you saw their faces when after a long way they achieved something, perhaps is there something that could be done now and that has something of the same feeling? Not so strong, perhaps, but still...?" Alternatively, an exercise might be set up, bringing the client back to a typical appetitive situation from his experience as a teacher to see if he can contact some of this in imagery. Then, the therapist might ask: "If you could take that feeling, that sense of creativity and intimacy with your students and move it to some area of your life now, where do you want to bring it? Also, the wife can be brought into imagery, making her give him advice on how to live the kind of life they valued together.

A possible way for the therapist to move from the bottom of the hierarchy and upwards would be to start in small events in the client's present life that seem to carry some meaning to the client. Perhaps the therapist notices something in some specific episode the client mentions. So, noticing something in the here and now, the therapist might focus on this saying: "I notice that this (something just mentioned) makes you look a bit interested...?" or: "There is a certain light in your eyes," or: "Something happens to you when you tell me about the phone call from your grandson. He seems to be important to you..." All of these would be examples of moving from "the bottom - up."

At the same time, as this work with building hierarchical functions of meaningful directions continues, the therapist needs to keep an eye on barriers turning up in this very process. This is natural, as imagining alternative ways of acting to an extent is an analog of actually acting that way. Furthermore, that is the very difficult thing to do for the client in his situation. When this occurs, the therapist should make it explicit in the interaction and use it to go back to the other strategies already covered.

Finally, but not least, we point out to the relevance of enveloping the clinical interactions hierarchically in a context of validation and comprehension.

In this part of the chapter, we have pointed out some relevant aspects of the process that establish hierarchical responding through MET. We have emphasized that functional analysis is a continuous activity that permeates all the therapeutic process and how it can be conducted with a focus on hierarchical responding even when different aspects of the operant are worked with. This operant is defined by the hierarchical function of personal meanings that, in the end, envelop any single action. We have tried to make the basic relational processes somehow transparent, allowing the identification of the relational process involved in building a repertoire of flexibility in the context of the particular coherence defining the inflexibility repertoire, the only repertoire dominating the scene when the therapy begins.

\section{Empirical evidence}

This part of the chapter is dedicated to pinpointing relevant empirical evidence about the core aspects presented in this chapter. Some of the studies have been referenced throughout the chapter, but now we will mention them briefly.

\subsection{Evidence of the transformation of eliciting and avoidance functions}

A number of studies have demonstrated the transformation of eliciting and avoidance functions. Dougher, Auguston, Markham, Greenway, and Wulfert (1994) showed the transfer of respondent eliciting and extinction functions through equivalence relations, whereas Auguston and Dougher (1997) demonstrated the transfer of avoidance evoking functions. These studies have been replicated and extended throughout the last two decades (e.g., Dougher, Hamilton, Fink, \& Harrington, 2007; Dymond, Schlund, Roche, \& Whelan, 2014; Luciano et al., 2014; RodríguezValverde, Luciano, \& Barnes-Holmes, 2009; RuizSánchez, Luciano, \& Rodriguez, submitted). 


\subsection{Evidence of the transformation of functions through hierarchical relations}

Gil et al. $(2012,2014)$ demonstrated the bottomup transformation of functions through hierarchical networks that included relations of coordination and distinction. As well, recent research has shown that class-inclusion responses can be trained through a nonarbitrary MET in autistic individuals (ZagrabskaSwiatkowska, Mulhern, Ming, Stewart, \& McElwee, 2020). Finally, two very recent studies have systematically demonstrated the contextual control in hierarchical responding at two levels (Callejón, 2020; Villarroel, Luciano, \& Ruiz-Sánchez, 2021). Firstly, they have demonstrated the top-down and bottom-up transformation of functions and, secondly, that the functions at the top dominate over the others. These data are extraordinarily relevant as analogies of the formation of the selfing behaviors and the domination of some functions over others.

\subsection{Evidence of the hierarchical organization of self-contents.}

Some preliminary studies have shown that selfcontents tend to be organized in hierarchical networks. For instance, Gil-Luciano, CalderónHurtado, Tovar, Sebastián, and Ruiz (2019) recruited 100 undergraduates who underwent several assessment phases, including a diagnostic interview, emotional symptoms and repetitive-negative-thinking (RNT), and a list of self-contents that usually serve as triggers for RNT. Participants selected the selfcontents they usually experienced and rated how much they engaged in RNT. Subsequently, they were provided with three diagrams showing how these self-contents could be organized: Coordination, Comparison, and Hierarchy. Eighty percent of participants organized their self-contents in hierarchical networks.

\subsection{Evidence of the pernicious effect of the relational functional class of responding in coordination with the functions of private events.}

There is a wide range of evidence that responding to eliminate painful private experiences (i.e., experiential avoidance) leads to increased suffering and preventing actions connected to hierarchical appetitive functions (i.e., values; Boulanger, Hayes, \& Pistorello, 2010). In the experimental arena, Hayes, Bissett, et al. (1999) used the cold pressor task to compare the effect of a 90minute acceptance protocol versus a control protocol.
Participants who received the acceptance protocol kept the arm immersed in ice water significantly longer than participants receiving the protocol promoting discomfort control. Using electric shocks as aversive stimulation, Gutiérrez, Luciano, Rodríguez, and Fink (2004) compared the effect of 20-min acceptance-based versus control-based protocols, both in the context of a personal value. Participants who received the acceptance-based protocol showed significantly greater pain tolerance and lower pain believability (i.e., assessment of maximal discomfort while participants continued on task) than participants in the control condition. The latter study was replicated and extended in further studies (e.g., McMullen et al., 2008; Páez-Blarrina et al., 2008).

\subsection{Evidence of repetitive negative thinking as a predominant response in coordination with aversive private events}

Robust experimental evidence has demonstrated that worry and rumination usually prolongs and extends aversive functions (e.g., see reviews in Ehring \& Watkins, 2008; Newman \& Llera, 2011), and leads to other forms of experiential avoidance such as alcohol consumption, binge eating,, selfinjury, and suicidal behavior. Experimental analogues of such problematic responding has been done in Gil-Luciano et al. (submitted) in the context of more and less problematic thoughts.

\subsection{Evidence of hierarchically framing ongoing behavior as a central relational process}

In a preliminary study with at-risk adolescents, Luciano et al. (2011) analyzed the differential effect of two defusion protocols. The first protocol involved MET in framing ongoing private events through deictic framings (I-Here, my private events-There). The second protocol also added hierarchical framings and interactions to promote motivational functions to the verbal discrimination of private events (i.e., appetitive augmental functions). The results showed that the second protocol had a greater effect on reducing the frequency of problematic behaviors and psychological inflexibility at the 4-month follow-up. Some experimental analogs have replicated the results of the previous study with different dependent variables: experimentally induced emotional distress (Foody, Barnes-Holmes, Barnes-Holmes, \& Luciano, 2013), tolerance on the cold pressor task and in the viewing of aversive films (Gil-Luciano, Ruiz, 
Valdivia-Salas, \& Suárez-Falcón, 2017), and the performance in cognitive demanding tasks (LópezLópez \& Luciano, 2017). In conclusion, these studies suggest that including explicit hierarchical framings between the individual and their private events and providing the hierarchical context of regulatory functions to this discrimination enhances the efficacy of the MET in contrast to incorporating only deictic framing. The latter is typical in many defusion exercises and the former of a consistent intervention to promote the self-as-context linked to valued functions.

Two more complex and unpublished studies have advanced over the previous research. GilLuciano, Tovar, Calderón-Hurtado, Sebastián, and Ruiz (submitted) tested the differential effect of two defusion protocols, one consisting of MET in deictic framing versus another one consisting of MET in deictic and hierarchical framing plus motivational functions. These protocols were directed either to the hierarchical aversive self-content or to an aversive self-content in a low-level of the hierarchy of self contents. The results showed that the most complete protocol was more effective than the remaining combinations and a control condition in reducing the detrimental effects of a rumination-induction procedure. Finally, López-López (2016) tested the efficacy of four defusion protocols in improving the performance on a cognitive demanding task (Protocol I: MET in deictic framing, Protocol II: MET in deictic + hierarchical framing, Protocol III: deictic + hierarchical framing + motivational functions, Protocol: deictic + hierarchical framing + motivational functions + hierarchically framing the chosen behavior). The results indicated that all the experimental protocols obtained a greater reduction in reported discomfort than the control condition. However, it was Protocol IV that obtained the greatest reduction in distress and only Protocols III and IV improved significantly performance in the cognitive demanding task.

\subsection{Evidence of contacting hierarchical appetitive functions as a central relational process in ACT}

Multiple experimental analogs have shown the effect of introducing a valued context in the protocols, either as the sole intervention strategy or as multi-component protocols (e.g., Gutiérrez et al., 2004; Hebert, Flynn, Wilson, \& Kellum, 2021; PáezBlarrina et al., 2008). Additionally, Luciano et al. (2010) found that aversive stimulation was rated as less intense when the discomfort was framed in hierarchy with advancing toward a valued direction (i.e., feeling discomfort and focusing on the task) than when framing in opposition.

Finally, only a few studies have analyzed the effect of protocols in the context of the transformation of functions as an experimental analog of the avoidance operant. For example, in Luciano et al. (2014), participants showed the acquisition of respondent eliciting and avoidance functions and their transference through equivalence classes. Afterward, the effect of a motivational protocol, that established a conditional relation between approaching previously avoided stimuli and a general value, was compared to a defusion protocol that included personally meaningful examples of approaching fear and a defusion exercise including both deictic and hierarchical framings. The latter protocol eliminated the experimentally induced avoidance responding in all participants, even in the presence of respondent activation, whereas the former condition was effective only in $30 \%$ of participants. Lastly, Ruiz-Sánchez, Luciano, and Rodríguez-Valverde (submitted) measured the derived rules during the whole process and analyzed the effect of an if -then motivational protocol as keeping in the task and receiving an specific consequence, versus a hierarchical motivation protocol in which approaching previously avoided stimuli and receiving shocks/noises were contextualized under personal meaning as a positive hierarchical reinforcer. The latter protocol was radically more effective than the former protocol in all cases. Thus, this study shows that motivation based on positive hierarchical reinforcers produces almost complete suppression of avoidance behavior in contrast to reduced levels of suppression when only the if-then motivational protocol.

\subsection{Evidence of the components of metaphors that increase their therapeutic effect}

Some experimental analogs have been conducted regarding the relational processes involved in metaphors in the clinical setting. Sierra, Ruiz, Flórez, Riaño-Hernández, and Luciano (2016) found that participants who received metaphors including common physical properties with the participants' pain showed a higher increase in pain tolerance on a cold pressor task than participants who received the same metaphors but without common physical properties. These data were replicated by Criollo, 
Díaz-Muelle, Ruiz, and García-Martín (2018). All these results were in line with more basic studies conducted by Ruiz and Luciano (2015) regarding the effect of common physical properties in analogy aptness and derivation.

Peña-Vargas et al. (submitted) analyzed the effect on pain tolerance of metaphors that include hierarchical relations between the participants and their pain and/or amplify the long-term consequences of flexible and inflexible behavior. The results showed that the metaphor that included both components was significantly more effective. Lastly, Ramírez, Ruiz, Peña-Vargas, and Bernal (submitted) analyzed the differential effects of presenting the metaphor by asking the individual to imagine herself as the protagonist of the story versus presenting the metaphor in the third person (Self vs. Other) and/or prompting the relational elaboration of the rules derived from the metaphor (Elaboration vs. No Elaboration). The results showed that including the components Self and Elaboration together significantly increased the participants' pain tolerance.

\subsection{Efficacy of brief interventions inspired in the RFT conceptualization of psychological flexibility as hierarchically framing ongoing behavior}

We will finish this section by highlighting some of the studies that have analyzed the effect of brief ACT protocols that explicitly define many of the relational processes of psychological flexibility (Törneke et al., 2016). Ruiz et al. (2016) tested the efficacy of a 1-session, repetitive-negative-thinking focused ACT protocol in a multiple-baseline design across 11 participants suffering from mild to moderate emotional disturbance. The results showed significant reductions in worry and rumination, emotional symptoms, experiential avoidance, cognitive fusion, and increased valued living in most participants. Ruiz et al. (2018) found very large effect sizes in the previous dependent variables when applying a 2-session of a similar protocol to participants with moderate emotional disorders. These initial promising findings were followed by a randomized controlled trial comparing a 2-session of the previous protocol against a waitlist control condition in participants with primary diagnoses of depression and/or generalized anxiety disorder. Again, a similar protocol produced large effect sizes and a high proportion of clinically significant changes (Ruiz, Peña-Vargas, et al., 2020).

\section{In concluding.}

In this chapter, we have invited to the reader to travel with us across different sites concerning the development of human suffering and its treatment from a functional analytic orientation. In this travel, we have used a boat under the title of hierarchical responding, a boat that has been used for several aims.

To summarize, we have used hierarchical responding as the final common path to understand the many relevant aspects of selfing behaviors. And we have used hierarchical responding as the final common path to identify the many faces of the therapeutic process towards building a repertoire that will allow the clients to live a meaningful life. We are aware of the interpretative conceptualization that some parts of the chapter involve. At the same time, we think there is the experimental evidence that has been signaling this path for years. Still, there is a lot of extraordinary and creative work needed to open the many closed doors-including ones that we do not even know about. Perhaps, the evolution of language has allowed us to learn such a highly flexible relational responding that allows us to integrate the efforts done by those who preceded us and integrate the many questions that we will leave behind. Perhaps this is why we often describe hierarchical responding at the top of our language abilities. 


\section{References}

Barnes-Holmes, D., Barnes-Holmes, Y., Luciano, C., \& McEnteggart, C. (2017). From the IRAP and REC model to a multi-dimensional multi-level framework for analyzing the dynamics of arbitrarily applicable relational responding. Journal of Contextual Behavioral Science, 6, 434-445.

Barnes-Holmes, D., Hayes, S. C., Dymond, S., \& O'Hora, D. (2002). Multiple stimulus relations and the transformation of stimulus functions. In S. C. Hayes, D. Barnes-Holmes, $\&$ B. Roche (Eds.), Relational frame theory (pp. 51-71). New York: Kluwer Academic.

Barnes-Holmes, D., Hayes, S.C., \& Dymond, S. (2001). Self and self-directed rules. In S. C. Hayes, D. Barnes-Holmes, \& B. Roche (Eds.), Relational frame theory (pp. 119140). New York: Kluwer Academic.

Boulanger, J. L., Hayes, S. C., \& Pistorello, J. (2010). Experiential avoidance as a functional contextual concept. In A. M. Kring, \& D. M. Sloan (Eds.), Emotion regulation and psychopathology: A transdiagnostic approach to etiology and treatment (pp. 107136). NY: Guilford Press.

Callejón, Z (2020). Training the hierarchical response. Thesis defended at University Almería, March 2020.

Criollo, A. B., Díaz-Muelle, J. S., Ruiz, F. J., \& García-Martín, M. B. (2018). Common physical properties improve metaphor effect even in the context of multiple exemplars. The Psychological Record, 68, 513-523.

Day, W. F. (1971). Humanistic psychology and contemporary behaviorism. Humanist, 31, 13-16.

Dougher, M. J., Augustson, E., Markham, M. R., Greenway, D. E., \& Wulfert, E. (1994). The transfer of respondent eliciting and extinction functions through stimulus equivalence classes. Journal of the Experimental Analysis of Behavior, 62, 331-351.

Dougher, M. J., Hamilton, D., Fink, B., \& Harrington, J. (2007). Transformation of the discriminative and eliciting functions of generalized relational stimuli. Journal of the Experimental Analysis of Behavior, 88, 179197.

Dougher, M. J., \& Hayes, S. C. (2000). Clinical behavior analysis. In M. J. Dougher (Ed.),
Clinical Behavior Analysis (pp. 11-25).

Reno, NV: Context Press.

Dymond, S., Schlund, M. W., Roche, B., \& Whelan, R. (2014). The spread of fear: Symbolic generalization mediates graded threatavoidance in specific phobia. Quarterly Journal of Experimental Psychology, 67, 247-259.

Ehring, T., \& Watkins, E. R. (2008). Repetitive negative thinking as a transdiagnostic process. International Journal of Cognitive Therapy, 1, 192-205.

Ferster, C. B. (1972). An experimental analysis of clinical phenomena. The Psychological Record, 22, 1-16.

Foody, M., Barnes-Holmes, Y., Barnes-Holmes, D., \& Luciano, C. (2013). An empirical investigation of hierarchical versus distinction relations in a self-based ACT exercise. International Journal of Psychology and Psychological Therapy, 13, 373-388.

Gil, E., Luciano, C., Ruiz, F. J., \& Valdivia-Salas, S. (2012). A preliminary demonstration of transformation of functions through hierarchical relations. International Journal of Psychology and Psychological Therapy, 12, 1-19.

Gil, E., Luciano, C., Ruiz, F. J., \& Valdivia-Salas, S. (2014). Towards a functional analysis of hierarchical classification: A further experimental step. International Journal of Psychology and Psychological Therapy, 14, 137-153.

Gil-Luciano, B., Calderón-Hurtado, T., Tovar, D., Sebastián-Sánchez, B., \& Ruiz, F. J. (2019). How are triggers for repetitive negative thinking organized? A relational frame analysis. Psicothema, 31, 53-59.

Gil-Luciano, B., Ruiz, F. J., Valdivia-Salas, S., \& Suárez-Falcón, J. C. (2017). Promoting psychological flexibility on tolerance tasks: Framing behavior through deictic/hierarchical relations and specifying augmental functions. The Psychological Record, 67, 1-9.

Gutiérrez, O., Luciano, C., Rodríguez, M., \& Fink, B. (2004). Comparison between an acceptancebased and a cognitive-control-based protocol for coping with pain. Behavior Therapy, 35, 767-783. 
Hayes, S. C. (1984). Making sense of spirituality. Behaviorism, 12, 99-110.

Hayes, S. C., Barnes-Holmes, D., \& Roche, B. (2001). Relational frame theory. New York: Kluwer Academic.

Hayes, S. C., Bissett, R., Korn, Z., Zettle, R. D., Rosenfarb, I., Cooper, L., \& Grundt, A. (1999). The impact of acceptance versus control rationales on pain tolerance. The Psychological Record, 49, 33-47.

Hayes, S. C., Strosahl, K. D., \& Wilson, K. G. (2012). Acceptance and commitment therapy. The process and practice of mindful change. New York: Guilford Press.

Herbert, E. R., Flynn, M. K., Wilson, K. G., \& Kellum, K. K. (2021). Values intervention as an establishing operation for approach in the presence of aversive stimuli. Journal of Contextual Behavioral Science.

Kohlenberg R. J., \& Tsai M. (1991). Functional analytic psychotherapy: Creating intense and curative therapeutic relationships. New York: Plenum Press.

López-López, J. C. (2016). Experimental analysis of defusion and its impact on performance.

Doctoral dissertation, Universidad de Almería.

López-López, J. C., \& Luciano, C. (2017). An experimental analysis of defusion interactions based on deictic and hierarchical framings and their impact on cognitive performance. The Psychological Record, 67, 485-497.

Luciano, C. (2017). The self and responding to the own's behavior. Implications of coherence and hierarchical framing. International Journal of Psychology and Psychological Therapy, 17, 3, 267-275.

Luciano, C., Gil-Luciano, B., Barbero, A., \& MolinaCobos, F. (2020). Perspective taking, empathy, and compassion. In M. Fryling, R. A. Rehfeldt, J. Tarbox, \& L. J. Hayes (Eds), Applied behavior analysis of language and cognition (pp. 281-300). Oakland, CA: Context Press.

Luciano, C., Molina, F., Gutiérrez-Martínez, O., Barnes-Holmes, D., Valdivia-Salas, S., Cabello, F., ... \& Wilson, K. G. (2010). The impact of acceptance-based versus avoidance-based protocols on discomfort. Behavior Modification, 34, 94-119.
Luciano, C., Ruiz, F. J., Gil-Luciano, B., \& MolinaCobos, F. J. (2021). Terapias contextuales. En E. Fonseca-Pedrero (Ed.), Manual de tratamientos psicológicos. Adultos (167199). Madrid: Pirámide.

Luciano, C., Ruiz, F. J., Vizcaíno-Torres, R., Sánchez, V., Gutiérrez-Martínez, O., \& López-López, J. C. (2011). A relational frame analysis of defusion interactions in Acceptance and Commitment Therapy. A preliminary and quasi-experimental study with at-risk adolescents. International Journal of Psychology and Psychological Therapy, 11, 165-182.

Luciano, C., Valdivia-Salas, S., Berens, N. M., Rodríguez-Valverde, M., Mañas, I. y Ruiz, F. J. (2009). Acquiring the earliest relational operants. Coordination, difference, opposition, comparison, and hierarchy. In R. A. Rehfeldt, \& Y. Barnes-Holmes (Eds.), Derived relational responding. Applications for learners with autism and other developmental disabilities (pp. 149-170). Oakland, CA: New Harbinger.

Luciano, C., Valdivia-Salas, S., Cabello, F., \& Hernández, M. (2009). Developing selfdirected rules. In R. A. Rehfeldt, \& Y. Barnes-Holmes (Eds.), Derived relational responding. Applications for learners with autism and other developmental disabilities (pp. 335-351). Oakland, CA: New Harbinger. Luciano, C., Valdivia-Salas, S., Ruiz, F. J.,

Rodríguez-Valverde, M., Barnes-Holmes, D., Dougher, M. J., ... \& Gutierrez-Martínez, O. (2014). Effects of an acceptance/defusion intervention on experimentally induced generalized avoidance: A laboratory demonstration. Journal of the Experimental Analysis of Behavior, 101, 94-111.

McMullen, J., Barnes-Holmes, D., Barnes-Holmes, Y., Stewart, I., Luciano, C., \& Cochrane, A. (2008). Acceptance versus distraction: Brief instructions, metaphors and exercises in increasing tolerance for self-delivered electric shocks. Behaviour Research and Therapy, 46, 122-129.

Páez-Blarrina, M., Luciano, C., Gutiérrez-Martínez, O., Valdivia, S., Ortega, J., \& RodríguezValverde, M. (2008). The role of values with personal examples in altering the functions of pain: comparison between acceptance-based 
and cognitive-control-based protocols. Behaviour Research and Therapy, 46, 84-97. Rodríguez-Valverde, M., Luciano, C., \& BarnesHolmes, D. (2009). Transfer of aversive respondent elicitation in accordance with equivalence relations. Journal of the Experimental Analysis of Behavior, 92, 85111.

Ruiz, F. J., Flórez, C. L., García-Martín, M. B., Monroy-Cifuentes, A., Barreto-Montero, K., García-Beltrán, D. M., Riaño-Hernández, D., Sierra, M. A., Suárez-Falcón, J. C., CardonaBetancourt, V., \& Gil-Luciano, B. (2018). A multiple-baseline evaluation of a brief acceptance and commitment therapy protocol focused on repetitive negative thinking for moderate emotional disorders. Journal of Contextual Behavioral Science, 9, 1-14.

Ruiz, F. J., \& Luciano, C. (2015). Common physical properties among relational networks improve analogy aptness. Journal of the Experimental Analysis of Behavior, 103, 498510.

Ruiz, F. J., Peña-Vargas, A., Ramírez, E. S., SuárezFalcón, J. C., García-Martín, M. B., GarcíaBeltrán, D. M., Henao, A. M., MonroyCifuentes, A., \& Sánchez, P. D. (2020). Efficacy of a two-session repetitive negative thinking-focused acceptance and commitment therapy (ACT) protocol for depression and generalized anxiety disorder: A randomized waitlist control trial. Psychotherapy, 57, 444-456.

Ruiz, F. J., Riaño-Hernández, D., Suárez-Falcón, J. C., \& Luciano, C. (2016). Effect of a onesession ACT protocol in disrupting repetitive negative thinking. International Journal of Psychology and Psychological Therapy, 16, 213-233.

Sierra, M. A., Ruiz, F. J., Flórez, C. L., RiañoHernández, D., \& Luciano, C. (2016). The role of common physical properties and augmental functions in metaphor effect. International Journal of Psychology and Psychological Therapy, 16, 265-279.

Skinner, B. F. (1945). The operational analysis of psychological terms. Psychological Review, 52, 270-276.

Skinner, B. F. (1953). Science and human behavior. New York: Macmillan.
Törneke, N. (2010). Learning RFT. An introduction to relational frame theory and its clinical application. Oakland, CA: New Harbinger.

Törneke, N. (2017). Metaphor in practice: A professional's guide to using the science of language in psychotherapy. Oakland, CA: New Harbinger.

Törneke, N. (2021). Clinical functional analysis and the process of change. Perspectivas em Análise do Comportamento, 12, 1-19.

Törneke, N., Luciano, C., Barnes-Holmes, Y., \& Bond, F. W. (2016). RFT for Clinical Practice. In R. D. Zettle, S. C. Hayes, D. Barnes-Holmes, \& A. Biglan (Eds.), The Wiley Handbook of Contextual Behavioral Science (pp. 254-272). New York: Wiley.

Villarroel, J., Luciano, C., \& Ruiz-Sánchez, L. J. (2021). Experimental analysis of hierarchical responding. Paper presented at ACBS Virtual World Conference.

Zagrabska-Swiatkowska, P., Mulhern, T., Ming, S., Stewart, I., \& McElwee, J. (2020). Training class inclusion responding in individuals with autism: Further investigation. Journal of Applied Behavior Analysis, 53, 2067-2080. 